Original Research Paper

\title{
$q$-Bernoulli's Equation
}

\author{
${ }^{1}$ Salih Y. Arbab and ${ }^{2,3}$ Sami H. Altoum \\ ${ }^{l}$ Department of Civil Engineering, Engineering College, Albaha University, KSA \\ ${ }^{2}$ Department of Mathematics, AL-Qunfudhah University College, Umm Al-Qura University, KSA \\ ${ }^{3}$ Academy of Engineering Sciences - Sudan
}

\author{
Article history \\ Received: 05-08-2020 \\ Revised: 26-09-2020 \\ Accepted: 02-10-2020 \\ Corresponding Author: \\ Sami H. Altoum \\ Department of Mathematics, \\ AL-Qunfudhah University \\ College, Umm Al-Qura \\ University, KSA and \\ Academy of Engineering \\ Sciences - Sudan \\ Email: shtoum@uqu.edu.sa
}

\begin{abstract}
The work shows the $q$-deformation of Bernoulli's equation, $q$ derivative and $q$-calculus are used to form a $q$-analogous of Bernoulli's equation. We introduce the theorem of $q$-Bernoulli's equation.
\end{abstract}

Keywords: $q$-Derivative, $q$-Calculus, Bernoulli's Differential Equation

\section{Introduction}

Historically, (Rguigui, 2015) solved a first nonlinear order ordinary differential equation:

$\frac{d x}{d t}+\alpha(t) y=\beta(t) y^{k}$

where, $\alpha(t)$ and $\beta(t)$ are differentiable functions and $k \in \mathbb{R}$ is fixed. The Equation (1) is called Bernoulli's equation, it has applications in mathematics, physics and engineering because it's non-linear differential equations and by substitution the Equation (1) becomes a linear with exact solutions. Bernoulli's equation is very important generally in various fields. Bernoulli improved his concept form his monitoring on liquids and his equation is fair only to incompressible fluids and study compressible fluids up to closely Mach number. It is probably to use the basic general rules of physics to improve same equations relevant to compressible fluids. There are various equations, each one adopt for a specific application, but whole are similar to Bernoulli's equation and all depend on the basic general rules of physics such as Newton's laws of motion or the first law of thermodynamics. Bernoulli's equation is occasionally valid for the flow of gases: As long as there is no transfer of kinetic or potential energy from the gas flow to the compression or expansion of the gas. In case the gas pressure and volume change together, then work will be done on or by the gas. In this situation, Bernoulli's equation in its incompressible flow form cannot be assumed to be useful. However, if the gas process is completely isobaric, or isochoric, then no work is done on or by the gas, so the simple energy balance is not disturbed. Consequently to the gas law, an isobaric or isochoric process is generally the only way to prove constant density in a gas. In mathematics, a $q$-analogues of a theorem, is identify or expressed a generalization that covering a new parameter $q$ that finding the basic theorem, identity or expression in the limit as $q \rightarrow 1$. In the most cases mathematicians are interested in $q$ analogues that arise basically, rather than in arbitrarily formulate of $q$-analogues of definite results. The earliest $q$-analogues studied in specifics of the basic hypergeometric series, which was presented in the 19th century. $q$-analogues are most commonly studied in the mathematical fields of special functions and combinatory. In these situations, the limit $q \rightarrow 1$ is often formal, as $q$ is often discrete-valued, it may represent as a prime power. $q$-analogues has applications in a number of fields, involving the study of fractals and multi-fractal measures and terms for the entropy of chaotic dynamical systems. The relation between fractals and dynamical systems results as due to many fractal patterns have the symmetries of Fuchsian groups in general as the modular group in particular. The relationship between hyperbolic geometry and ergodic theory, where the elliptic integrals and modular forms play a basic role; the $q$-series themselves are approximately related to elliptic integrals. $q$-analogues also can be seen in the study of quantum 
groups and in $q$-deformed super algebras (Abdi, 1962; Adams, 1928; Gasper and Rahman, 1990; Jackson, 1910; Leeuwen and Maassen, 1995). The $q$-calculus and $q$ derivative have applications in number of areas such as mathematics, hyperbolic geometry, number theory, physics, engineering and quantum calculus. Rguigui (2018b) reformed $q$-Euler equation, (Alanazi et al., 2020) expanded that the falling body problem and pointed that the exact solutions acquired by using $q$ calculus decreased to the corresponding solutions in classical mechanics when the quantum parameter $q$ approach to one. Kac and Cheung (2001) introduced a $q$ analog of classical derivative of binomial theorem and Taylor's theorem. Adams (1928) solved transonic gas equation by using $q$-derivative and show that the huge different between classical solution and a new $q$ analogous solution of transonic gas equation. Akça et al. (2019) solved many differential equations by using $q$ calculus. This article issued by follows. In section 2 we give some notations and definitions of $q$-calculus, section 3 includes the main principles of $q$-derivative. Finally, section 4 introduced $q$-deformation of Bernoulli's equation by using $q$-derivative.

\section{$q$-Calculus}

In this part, some notations and definitions on the qcalculus are submitted.

\section{Definition 2.1}

A $q$-analogues of a number is define by:

$$
\begin{aligned}
& {[n]_{q}=\frac{q^{n}-1}{q-1}=1+q+q^{2}+\ldots+q^{n-1},[0]_{q}=0 .} \\
& \lim _{n \rightarrow+\infty}[n]_{q}=\lim _{n \rightarrow+\infty} \frac{q^{n}-1}{q-1}=\frac{1}{1-q}=[\infty]_{q},|q|<1
\end{aligned}
$$

\section{Definition 2.2}

A $q$-Factorial of natural number $[n]_{q}$ ! is define by:

$$
[n]_{q} !=\left\{\begin{array}{cc}
{[n]_{q} \times[n-1]_{q} \times \ldots \times[3]_{q} \times[1]_{q},} & n=1,2, \ldots \\
1 & n=0 .
\end{array}\right.
$$

\section{Definition 2.3}

A $q$-Binomial coefficients is define by:

$$
\left[\begin{array}{l}
n \\
k
\end{array}\right]=\frac{[n]_{q} !}{[k]_{q} ![n-k]_{q} !}
$$

\section{Definition 2.4}

A $q$-analogues of $(x-a)^{n}$ is defined by:

$$
(x-a)_{q}^{n}=\prod_{k=0}^{n-1}\left(x-q^{k} a\right)=\sum_{k=0}^{n}\left[\begin{array}{l}
n \\
k
\end{array}\right]_{q} q^{\frac{k(k-1)}{2}} a^{k} x^{n}-k .
$$

\section{$q$-Derivative}

The derivativation of a function $f$ with respect to $x$ is defined by:

$$
\frac{d f}{d x}=\lim _{h \rightarrow 0} \frac{f(x+h)-f(x)}{h}
$$

There are different definitions of the derivative of a function as $p$-Derivative and $q$-derivative. A $q$-derivative $D_{q} f$ of a function $\mathrm{f}$ is defined by:

$$
\left(D_{q} f\right)=\frac{f(x)-f(q x)}{(1-q) x}, x \neq 0
$$

And $\left(D_{q} f\right)(0)=f^{\prime}(0)$ provide $f^{\prime}(0)$ exists. The key concept is $q$-derivative operator defined as follows when $q \in(0,1)$ :

$$
\frac{d f}{d x}=\lim _{q \rightarrow 1}\left(D_{q} f\right)=\lim _{q \rightarrow 1} \frac{f(x)-f(q x)}{(1-q) x}
$$

if $\mathrm{f}$ is differentiable. For an example, we can calculate the $D_{q}\left(x^{3}\right)$ :

$$
D_{q}\left(x^{3}\right)=\frac{x^{3}-(q x)^{3}}{(1-q) x}=\left(1+q+q^{2}\right) x^{2}=3 x^{3}
$$

And:

$$
D_{q}\left(x^{n}\right)=\frac{x^{n}-(q x)^{n}}{(1-q) x}=\left(1+q+q^{2}+\ldots\right) x^{n-1}=[n]_{q} x^{n-1}
$$

If given $f, g$ is differentiable functions, then $D_{q}$ has the property of the linearity, that for any constants $a$ and $b$ :

$$
D_{q}(a f+b g)=a D_{q} f+b D_{q} g
$$

The $q$-derivative of product and the quotient rule applies:

$$
\begin{aligned}
& D_{q}(f(x) g(x))=f(x) D_{q} g(x)+g(q x) D_{q} f(x) \\
& D_{q}\left(\frac{f}{g}\right)(x)=\frac{g(q x) D_{q} f(x)-f(q x) D_{q} g(x)}{g(x) g(q x)}
\end{aligned}
$$

The product and quotient rule are modified but it closed to the ordinary product and quotient rule when $q \rightarrow 1$. 
There is no general chain rule for $q$-derivative, an exception is $u(x)=a x^{k}$ :

$$
D_{q} f(u(x))=\left(D_{q^{k}} f\right)(u(x)) D_{q} u(x) .
$$

For the higher order of $q$-derivative (Wang and Chu, 2018). It is known as:

$$
D_{q}^{2} f(x)=D_{q}\left(D_{q}\right) f(x)
$$

and:

$$
D_{q}^{n} f(x)=D_{q}\left(D_{q}^{n-1}\right) f(x) \text { for } n=2,3,4 \ldots
$$

with the form that $D_{q}^{0} f(x)=f(x)$ for the operation of identity. In general we preset the following explicit formula:

$$
D_{q, x}^{n} f(x)=x^{-n} \sum_{k=0}^{n} q^{k} \frac{\left(q^{-n} ; q\right)_{k}}{(q ; q)_{k}} f\left(q^{k} x\right)
$$

anywhere the $q$-shifted factorial of $x$ is presented by $(x$; $q)_{0} \equiv 1$ and:

$$
(x ; q)_{n}=\prod_{k=0}^{n-1}\left(1-x q^{k}\right) \text { for } n=1,2, \ldots
$$

\section{$q$-Deformation Bernoulli's Equation}

As an $q$-analogous of (1) we deduce a new formula of $q$-Bernoulli's equation as follows:

$$
D_{q} y+\alpha(t) y=\beta(t) y^{k}
$$

\section{Theorem 4.1}

For $q \in(0,1)$, the solution of the $q$-Bernoulli's equation is presented by:

$$
\begin{aligned}
& y(t)=y\left(q^{j} t\right) \\
& +\sum_{i=1}^{j} q^{j-1}(1-q)\left[-\alpha\left(q^{j-1} t\right) y\left(q^{j-1} t\right)+\beta\left(q^{j-1} t\right) y^{k}\left(q^{j-1} t\right)\right],
\end{aligned}
$$

\section{Proof}

Let $D_{q} y+\alpha(t) y=\beta(t) y^{k}$ then, the $q$-derivative with respect to $t$ is:

$$
\frac{y(t)-y(q t)}{(1-q) t}=-\alpha(t) y(t)+\beta(t) y^{k}(t)
$$

$$
\begin{aligned}
& y(t)-y(q t)=-t(1-q) \alpha(t) y(t)+t(1-q) \beta(t) y^{k}(t) \\
& y(q t)-y\left(q^{2} t\right)=-q t(1-q) \alpha(q t) y(q t)+q t(1-q) \beta(q t) y^{k}(q t) \\
& y\left(q^{2} t\right)-y\left(q^{3} t\right)=-q^{2} t(1-q) \alpha\left(q^{2} t\right) y\left(q^{2} t\right)+q^{2} t(1-q) \beta\left(q^{2} t\right) y^{k}\left(q^{2} t\right) \\
& y\left(q^{3} t\right)-y\left(q^{4} t\right)=-q^{3} t(1-q) \alpha\left(q^{3} t\right) y\left(q^{3} t\right)+q^{3} t(1-q) \beta\left(q^{3} t\right) y^{k}\left(q^{3} t\right) \\
& \quad \vdots \\
& y\left(q^{j-1} t\right)-y\left(q^{j} t\right)=-q^{j-1} t(1-q) \alpha\left(q^{j-1} t\right) y\left(q^{j-1} t\right)+q^{j-1} t(1-q) \beta\left(q^{j-1} t\right) y^{k}\left(q^{j-1} t\right)
\end{aligned}
$$

Thus:

$$
\begin{aligned}
& y(t)-y\left(q^{j} t\right)=\sum_{i=1}^{j} q^{j-1} t(1-q)\left[-\alpha\left(q^{j-1} t\right) y\left(q^{j-1} t\right)+\beta\left(q^{j-1} t\right) y^{k}\left(q^{j-1} t\right)\right] \\
& y(t)=y\left(q^{j} t\right)+\sum_{i=1}^{j} q^{j-1} t(1-q)\left[-\alpha\left(q^{j-1} t\right) y\left(q^{j-1} t\right)+\beta\left(q^{j-1} t\right) y^{k}\left(q^{j-1} t\right)\right]
\end{aligned}
$$

\section{Remark 1}

If $j \rightarrow \infty$ we deduce that:

$$
y(t)-y(0)+\sum_{i=1}^{\infty} q^{j-1} t(1-q)\left[-\alpha\left(q^{j-1} t\right) y\left(q^{j-1} t\right)+\beta\left(q^{j-1} t\right) y^{k}\left(q^{j-1} t\right)\right] .
$$




\section{Remark 2}

In this study we introduced the $q$-analogue Bernoulli's equation. A free analogue of some nuclear algebras of operators acting on space of holomorphic functions on a free analogue complexification of real nuclear space can be studied and we expect to develop a new quantum white noise analogue of free-Bernoulli's equation (Rguigui, 2015; Parker, 2013; Rguigui, 2016ab; 2018a-b; Altoum, 2018).

\section{Acknowledgment}

Our thanks and appreciations goes to referees for their cureful reading of our manscipt and helpful remarks.

\section{Author's Contributions}

All authors equally contributed in this work.

\section{Ethics}

This article is original and contains unpublished material. The corresponding author confirms that all of the other authors have read and approved the manuscript and no ethical issues involved.

\section{References}

Abdi, W. H. (1962). On certain q-difference equations and q-Laplace transforms. Proc. Natl. Acad. Sc. India A, 28, 1-15.

Adams, C. R. (1928). On the linear ordinary q-difference equation. Annals of mathematics, 195-205.

Akça, H., Benbourenane, J., \& Eleuch, H. (2019, November). The q-derivative and differential equation. In Journal of Physics: Conference Series (Vol. 1411, No. 1, p. 012002). IOP Publishing.

Alanazi, A. M., Ebaid, A., Alhawiti, W. M., \& Muhiuddin, G. (2020). The falling body problem in quantum calculus. Front. Phys. 8: 43. doi: $10.3389 /$ fphy.
Altoum, S. H. (2018). q-deformation of the square white noise Lie algebra. Transactions of A. Razmadze Mathematical Institute, 172(2), 133-139.

Gasper, G., \& Rahman, M. (1990). Encyclopedia of Mathematics and its Applications (Vol. 35, pp. 1-8). Cambridge: Cambridge university press.

Jackson, F. H. (1910). q-Difference equations. American Journal of Mathematics, 32(4), 305-314.

Kac, V., \& Cheung, P. (2001). Quantum calculus. Springer Science \& Business Media.

Leeuwen, H. V., \& Maassen, H. (1995). A q deformation of the Gauss distribution. Journal of mathematical physics, 36(9), 4743-4756.

Parker, A. E. (2013). Who Solved the Bernoulli Differential Equation and How Did They Do It? The College Mathematics Journal, 44(2), 89-97.

Rguigui, H. (2015). Quantum Ornstein-Uhlenbeck semigroups. Quantum Studies: Mathematics and Foundations, 2(2), 159-175.

Rguigui, H. (2016a). Characterization of the QWNconservation operator and applications. Chaos, Solitons \& Fractals, 84, 41-48.

Rguigui, H. (2016b). Wick differential and Poisson equations associated to the QWN-Euler operator acting on generalized operators. Mathematica Slovaca, 66(6), 1487.

Rguigui, H. (2018a). Fractional number operator and associated fractional diffusion equations. Mathematical Physics, Analysis and Geometry, 21(1), 1.

Rguigui, H. (2018b). Characterization theorems for the quantum white noise gross Laplacian and applications. Complex Analysis and Operator Theory, 12(7), 1637-1656.

Wang, X., \& Chu, W. (2018). Approach of q-Derivative Operators to Terminating q-Series Formulae. Communications in Mathematics, 26(2), 99-111. 